\title{
Fisiopatologia cirúrgica do hematoma putaminal
}

\author{
Marcos Augusto Stávale Joaquim¹, Gustavo Cartaxo Patriota ${ }^{1}$, André de Macedo Bianco² \\ Instituto de Neurociências, São Paulo, SP. \\ Serviço de Neurocirurgia do Hospital Nove de Julho, São Paulo, SP.
}

\section{RESUMO}

Os autores descrevem a fisiopatologia da hipertensão intracraniana relacionada aos hematomas putaminais espontâneos e a função da cirurgia no tratamento. O conhecimento da fisiopatologia desmistifica o resultado de muitas publicações prévias e torna óbvia a necessidade da operação nos casos em que se opta por tratar o paciente nos quais há hipertensão intracraniana.

\section{PALAVRAS-CHAVE}

Hemorragia cerebral. Hematomas intraparenquimatosos cerebrais. Hematomas putaminais.

\begin{abstract}
Putaminal hematoma. Surgical pathophysiology

The authors discuss the pathophysiology of the intracranial hypertension relative to putaminal or basal ganglia spontaneous hematomas. The role of the surgery is discussed. The current pathophysiological knowledge is strong enough to be the counterpart to the previous published statistical studies, becoming obvious the necessity of the surgical drainage when the patient is going to be treated and intracranial hypertension is present.
\end{abstract}

\section{KEY WORDS}

Intracerebral haemorrahge. Intracerebral haematomas. Putaminal haematomas.

\section{Introdução}

A hipertensão intracraniana (HIC) progressiva é fatal e sua progressão é exponencial segundo a curva de Langfitt. A interrupção precoce dessa ascensão é um procedimento salvador da vida. O tratamento da HIC começa por sua causa inicial; massas expansivas constituem, em geral, essa causa, não só pelo próprio volume como também pelo inchaço cerebral que causam pela vasodilatação isquêmica, pois aumentam primariamente a pressão intracraniana (PIC) e diminuem a pressão de perfusão encefálica (PPE), o que causa abertura da arteríola pré-capilar. Esse inchaço eleva o volume sanguíneo encefálico (VSE) e a PIC adicionalmente. ${ }^{1,10,13-16,18-20,22-24,26-29,31,41,42,48,49}$

As massas expansivas são tratadas com exérese cirúrgica, e os inchaços, com medidas de suporte intensivo que manipulam o VSE (elevação do decúbito, hiperventilação, manitol, barbitúricos e outros). 7,8,17,36,38,47 $\mathrm{O}$ uso de tais medidas na presença do hematoma que desencadeou a HIC e o inchaço tem efeito apenas transitório e prolonga o sofrimento isquêmico do encéfalo normal se a massa constituída pelo coágulo não for retirada de início. O inchaço isquêmico por vasodilatação e aumento do VSE será tanto maior quanto mais tempo persistir a HIC e a queda da PPE iniciais, e esta é a causa da falência da drenagem cirúrgica, quando a HIC volta a aumentar após a evacuação do coágulo na reperfusão de um leito vascular encefálico dilatado pela acidose láctica que ocorreu, secundária à queda da PPE e do fluxo sanguíneo encefálico (FSE) na presença do hematoma.

\section{Fisiopatologias das alterações pós-hemorrágicas}

Um sangramento pequeno não eleva a PIC e não decresce a PPE de maneira significativa e, portanto, não

1. Instituto de Neurociências, São Paulo, SP.

2. Serviço de Neurocirurgia do Hospital Nove de Julho, São Paulo, SP. 
há inchaço por vasodilatação isquêmica do encéfalo. As dimensões do coágulo não são isoladamente importantes, pois pacientes idosos ou alcoólatras com aumento ex-vácuo do compartimento liquórico aceitam maiores volumes de hematoma à medida que extruem líquido da caixa craniana. Há mais complacência intracraniana. Pacientes jovens, com menor compartimento liquórico, desenvolvem HIC com volumes menores de hematoma, pois a complacência intracraniana é menor. Uma massa hemática suficiente para elevar a PIC e decrescer a PPE certamente gerará isquemia, que obrigatoriamente se associa à abertura de arteríola pré-capilar e à vasodilatação encefálica. Esse aumento do VSE coloca o paciente na cascata vasodilatadora, e esta o coloca na fase exponencial ascendente da curva de Langfitt. A cascata vasodilatadora é um mecanismo progressivo, que se automantém e que eleva a PIC até causar a morte encefálica (Figura 1). O objetivo terapêutico é bloquear a cascata vasodilatadora, começando pelo bloqueio de sua causa, ou seja, pela retirada da massa hemática que causa isquemia encefálica por provocar a queda da PPE e do FSE. ${ }^{18,32}$

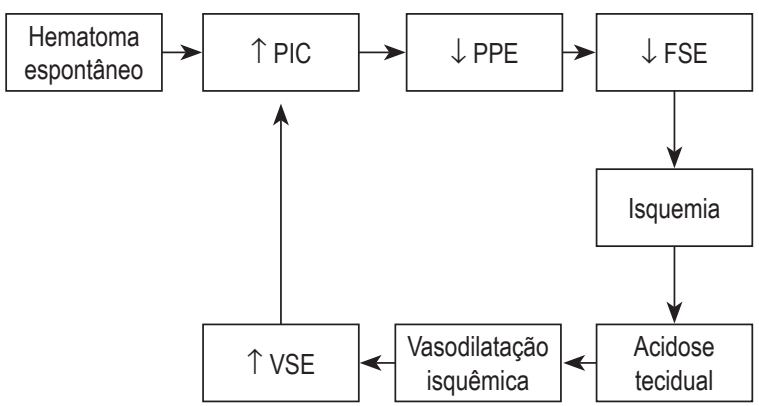

PIC: pressão intracraniana; PPE: pressão de perfusão encefálica; FSE: fluxo sanguíneo encefálico; VSE: volume sanguíneo encefálico.

Figura 1 - Cascata vasodilatadora no hematoma intracerebral espontâneo.

\section{Reperfusão após drenagem do hematoma}

As complicações hipervolêmicas encefálicas por reperfusão podem ser hiperêmicas (de alto fluxo) ou oligoêmicas (de baixo fluxo), que, na verdade, são fases evolutivas de um mesmo processo fisiopatológico. Após a retirada da massa expansiva a PPE é devolvida a um leito vascular dilatado pela acidose tecidual isquêmica. O aumento da pressão hidrostática na microcirculação causa uma hipervolemia e um inchaço encefálico a principio hiperêmico (de alto fluxo), ou seja, associado a um aumento do VSE e do FSE, enquanto a PIC não aumenta significativamente a ponto de decrescer a PPE. ${ }^{25,52}$

A hiperemia reperfusional pode ser autolimitada (hiperemia reativa transitória) ou pode ser progressiva se a acidose tecidual for grave. Na última situação, um aumento progressivo no VSE transforma um inchaço, que a princípio era hiperêmico, em um inchaço oligoêmico (de baixo fluxo). Esse fato ocorrerá quando a hipervolemia encefálica causar HIC que se associará à baixa PPE até a isquemia global estabelecida (Figura 2). Inchaços causam nova elevação da PIC após a drenagem dos hematomas e devem ser tratados precocemente e rigorosamente para controlar a cascata vasodilatadora, ou sequelas isquêmicas graves e até mesmo a morte encefálica poderão ocorrer. Assim, mais uma vez, mostrase a importância da descompressão interna (drenagem do hematoma) precoce. Ressalve-se que uma isquemia grave e persistente pode gerar o fenômeno da "não reperfusão" secundário à trombose da microcirculação e ao edema endotelial.

Quando um hematoma que produz HIC não é drenado, o inchaço por vasodilatação isquêmica progressiva ocorre já caminhando diretamente para a oligoemia, não havendo passagem pela fase hiperêmica reperfusional.

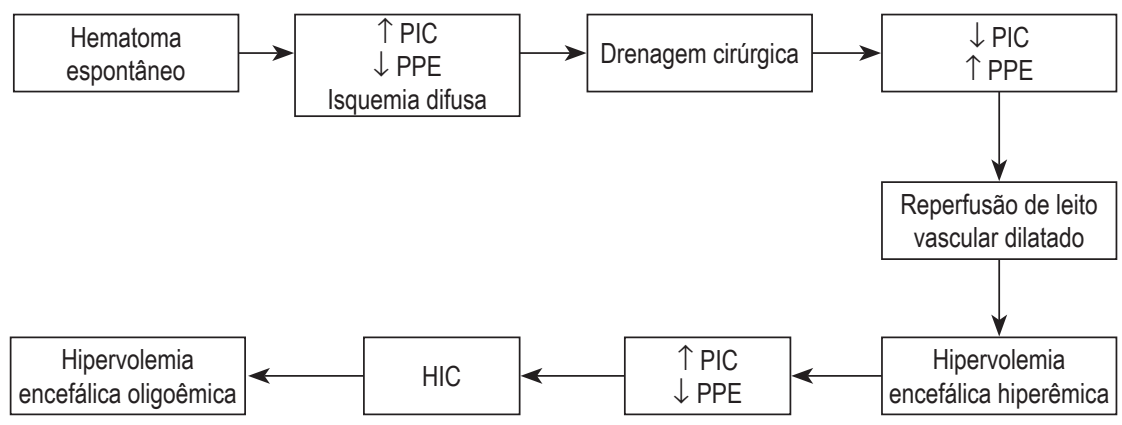

Figura 2 - Passagem do inchaço hiperêmico após a drenagem para o inchaço oligoêmico. 


\section{Eventos associados}

Hemorragia ventricular é drenada com ou sem o uso de endoscopia. A derivação ventricular externa é mantida para se tratar a hidrocefalia. Monitores intraventriculares de PIC são preferenciais. Lavagem ventricular com trombolíticos não é utilizada. Hemorragias ventriculares contralaterais são tratadas com a abertura do septo pelúcido ou com endoscopia transcortical. A hemorragia ventricular sempre foi considerada um sinal de mau prognóstico. Esse fato não piora diretamente o sofrimento do tecido nervoso, pois a invasão da cavidade ventricular apenas desloca liquor e não lesa neurônios. Assim, a piora do prognóstico deve-se à obstrução do fluxo liquórico e à hidrocefalia secundária que piora sobremaneira a HIC e acelera o sofrimento cerebral isquêmico pela queda adicional da PPE. Logo, a hemorragia ventricular deve ser objetiva e cirurgicamente tratada, e cateter ou monitor intraventricular inserido. $37,40,44,47$

\section{Fatores prognósticos}

Como citadas anteriormente, dimensões volumétricas do coágulo são importantes, e não apenas seu diâmetro. O estudo volumétrico deve ser realizado em relação ao volume do crânio e do espaço liquórico, particularmente em pacientes com atrofia encefálica e com grande compartimento liquórico e que resistem mais a aumentos da PIC. Em segundo lugar, a perda imediata da consciência reflete HIC aguda ou dissecção hemorrágica do tronco encefálico. A perda tardia ou progressiva da consciência implica HIC progressiva ou ressangramentos.

O tempo desde a hemorragia até a drenagem interfere no tempo de permanência da HIC e da isquemia encefálica e em suas consequências vasodilatadoras e causadoras do inchaço pelo aumento do VSE. Drenagens precoces associam-se a menores isquemias e inchaços isquêmicos e a melhores prognósticos. $\mathrm{O}$ mesmo raciocínio que é feito com relação à precocidade das descompressões externas em casos de infarto hemisférico maciço associado à HIC pode ser transportado para a descompressão interna (retirada do coágulo) em casos de hematoma putaminal. ${ }^{35,38,43,46}$

\section{Controle da pressão arterial e drenagem cirúrgica}

O hematoma putaminal é produto, em geral, de uma crise hipertensiva arterial sistêmica. A ruptura de um microaneurisma de Charcot-Bouchard nas artérias perfurantes do polígono de Willis o provoca.

Ao receber o doente na sala de emergência, nota-se grave hipertensão arterial sistêmica por dois motivos. O primeiro deve-se à crise de hipertensão arterial inicial. O segundo deve-se ao reflexo de Cushing, caracterizado pela elevação da pressão arterial sistêmica para manter a PPE. ${ }^{11,12}$

O controle da pressão arterial média (PAM) com hipotensores potentes pode interferir com o primeiro motivo e melhorar a condição hemorrágica, mas pode neutralizar o reflexo de Cushing e aumentar a isquemia cerebral, a vasodilatação isquêmica, o VSE, o inchaço e a PIC. Assim, o paciente deve ser imediatamente levado ao centro cirúrgico, submetido à monitorização da PIC, e operado se houver HIC. A partir de então a PAM, que já costuma cair abruptamente durante a cirurgia após a drenagem do hematoma, pode ser controlada desde que se mantenha uma PPE de aproximadamente $70 \mathrm{~mm}$ de $\mathrm{Hg}$.

\section{Acesso cirúrgico}

A craniotomia é centrada na sutura coronária, à frente da área motora, e a intrusão cortical dá-se ao nível da sutura coronária ou anteriormente a ela. Uma pequena craniotomia e uso do microscópio cirúrgico permitem drenagem, lavagem da cavidade e hemostasia adequada. Após a craniotomia pode haver hérnia cerebral externa, que costuma melhorar após a retirada do coágulo. A drenagem estereotáxica é uma alternativa, a nosso ver, menos segura e menos eficaz. ${ }^{2,30,45}$

A intrusão cortical é sempre alta, na superfície superior do hemisfério, e nunca lateral ou transcisternal por entre os ramos da artéria cerebral média, pois não há espaço de trabalho microcirúrgico em um encéfalo inchado, particularmente no hemisfério dominante, pois a área de Broca poderá ser lesada. Microscopia cirúrgica, sempre utilizada, ou a endoscopia podem demonstrar ótima observação interna da superfície da cavidade do hematoma. Os acessos transcisternais silvianos são absolutamente indesejáveis, pois se trabalha em um cérebro inchado e entre os ramos de $M_{1}$ a $M_{4}$ da artéria cerebral média e a possibilidade de lesão é óbvia.

\section{Contraposição à estatística}

Diversas publicações, antigas e recentes, consideram que a cirurgia não interfere no resultado e determinadas porcentagens dos doentes evoluem para 
o óbito. Essas publicações não levam em consideração a presença ou ausência de HIC, da queda da PPE, a complacência intracraniana, o volume do hematoma em relação ao compartimento liquórico cerebral ou o momento da cirurgia. Sequer monitorizam a PIC desses doentes, o que é de importância óbvia na decisão cirúrgica. Paradoxalmente, tais publicações são usadas ainda hoje na determinação das condutas prejudicando os doentes, neutralizando suas chances de sobrevivência e aumentando as sequelas. ${ }^{3,4,6,9,21,34,35}$

É óbvio que, se o volume do hematoma é suficiente para causar HIC e queda na PPE, uma vasodilatação arteriolar acidótica isquêmica aumentará o VSE no sistema de capacitância volêmico encefálico (microcirculação, vênulas e veias), e a cascata vasodilatadora secundária ocorrerá. Assim, hematomas maiores com quadro clínico e imagenológico de HIC devem ser imediatamente drenados e, após a cirurgia, a PIC é monitorizada, pois um inchaço pela reperfusão após a drenagem que restabelece a PPE no leito vascular dilatado pela acidose pode ocorrer. Tal inchaço será tanto maior quanto maior o tempo decorrido até a drenagem do coágulo. Assim, medidas paliativas de suporte intensivo, se usadas isoladamente no início e sem a drenagem do coágulo, embora possam controlar transitoriamente a situação, mantêm o sofrimento isquêmico e o inchaço, que poderão, pela acidose láctica tecidual progressiva, não mais responder a esses tratamentos e permitir a elevação exponencial da PIC. Pouco adianta manipular o VSE isquemicamente aumentado se o hematoma continua presente e se mostrou suficientemente volumoso para aumentar a PIC, isquemiar o encéfalo e aumentar o VSE. Ou seja, o volume do hematoma não diminuirá com as medidas de suporte intensivo, que apenas manipulam o VSE. Assim, o efeito de massa do coágulo deve ser removido e as medidas de suporte intensivo serão muito úteis se houver inchaço associado.

Pacientes portadores de hematomas de indicação cirúrgica discutível são imediatamente levados ao centro cirúrgico e um monitor de PIC é instalado. Se a PIC estiver baixa ( $<15$ ou $20 \mathrm{~mm}$ de $\mathrm{Hg}$ ), uma conduta clínica pode ser adotada. Se a PIC se elevar, a operação é realizada. Se a PIC estiver alta no momento da inserção do cateter ( $>20 \mathrm{~mm}$ de $\mathrm{Hg}$ ) ou se houver ondas patológicas tipo A, a cirurgia é imediatamente realizada e o cateter de monitorização da PIC mantido para orientar o suporte neurointensivo da eventual HIC pós-operatória secundária ao inchaço por vasodilatação, que pode ocorrer na reperfusão do encéfalo isquêmico e vasodilatado. É impossível tratar um paciente com HIC na UTI sem a monitorização da PIC. Inibidores do crescimento de hematomas menores como o Fator VII podem ser administrados durante o tratamento em certos pacientes. . $^{5,33,39}$

\section{Conclusão}

Não há lugar na neurociência contemporânea para o tratamento conservador de pacientes portadores de hematomas putaminais que apresentam HIC à admissão. A cirurgia com microscopia e tecnologia associada é indicada. Retardo equivocado na indicação da drenagem mantém a HIC mais tempo, piora a acidose tecidual e associa-se aos inchaços reperfusionais pós-operatórios por aumento do VSE cada vez mais graves. Estes podem causar graves reelevações da PIC, quedas da PPE e isquemia tecidual difusa que podem causar o estado vegetativo ou a morte encefálica.

\section{Referências}

1. Auer LM, Ishiyama N, Pucher R. Cerebrovascular response to intracranial hypetension. Acta Neurochir(Wien).1987;84:124-8.

2. Barret RJ, Hussain R, Coplin WN, Berry S, Keyl PM, Hanley $\mathrm{DF}$, et al. Frameless stereotactic aspiration and thrombolysis of spontaneous intracerebral hemorrhage. Neurocrit Care. 2005;3:237-45.

3. Batjer HH, Reisch JS, Allen BC, Plaizier LJ, Su CJ. Failure of surgery to improve outcome in hypertensive putaminal hemorrhage. A prospective randomized trial. Arch Neurol. 1990;47:1103-6.

4. Brandt L, Sonesson B, Ljunggren B, Saveland H. Ruptured middle cerebral artery aneurysm with intracerebral hemorrhage in young patients appearing moribund: emergency operation? Neurosurgery. 1987;20:925-9.

5. Brott T, Broderick J, Kothari R, Barsan W, Tomsick T, Sauerbeck L, et al. Early hemorrhage growth in patients with intracerebral hemorrhage. Stroke. 1997;28:11-5.

6. Cuatico W, Adib S, Gastón P. Spontaneous intracerebral hematomas. Surgical appraisal. J Neurosurg. 1964;22:56975.

7. Dereeper E, Berré J, Vandesteene A, Lefranc F, Vincent JL. Barbiturate coma for intracranial hypertension: clinical observations. J Crit Care. 2002;17:58-62.

8. Diringer MN. Intrarecebral hemorrhage: pathophysiology and management. Crit Care Med. 1993;21:1591-603.

9. Fayad PB, Awad IA. Surgery for intracerebral hemorrhage. Neurology. 1998;51:69-73.

10. Fearnside MR, Adams CBT. The treatment of raised intracranial pressure following aneurysm surgery. J Neurol Neurosurg Psychiatry. 1980;43:957-61.

11. Fitch W, Mc Dowall ED. Systemic vascular responses to increased intracranial pressure: 1. Effects of progressive intracranial epidural ballon expansion on intracranial pressure and systemic circulation. J Neurol Neurosurg Psychiatry. 1977;40:833-42.

12. Fitch W, McDowall DG, Keaney MP, Pickerodt VWA. Systemic vascular reponses to incrased intracranial pressure: 2. The "Cushing" response in the presence of intracranial space-occupying lesions: systemic and cerebral heamodinamic studies in the dog and the baboon. J Neurol Neurosurg Psychiatry. 1977;40:843-52.

13. Gray WJ, Rosner MJ. Pressure-volume index as a function of cerebral perfusion pressure. Part 2. The effects of low cerebral perfusion and autoregulation. J Neurosurg. 1987;67:377-80. 
14. Grubb RL, Paichle ME, Phelps ME, Patcheson PA. Effects of increased intracranial pressure in cerebral blood volume, blood flow and oxygen utilization in monkeys. J Neurosurg. 1975;43:385-98.

15. Hedges TF, Weinstein JD. Cerebrovascular responses to increased intracranial pressure. J Neurosurg. 1964;21:292-7.

16. Hekmatpanah J. Cerebral circulation and perfusion in experimental increased intracranial pressure. J Neurosurg. 1970;32:21-9

17. Hsieh PC, Awad IA, Getch CC, Bendok BR, Rosenblat SS, Batjer $\mathrm{HH}$. Current updates in perioperative management of intracerebral hemorrhage. Neurosug Clin North Am. 1978;19:401-4.

18. Janny P, Colnet G, Georget AM, Chazal J.Intracranial pressure with intracerebral hemorrhages. Surg Neurol. 1978;10:371-5.

19. Johnson IH, Rowan JO, Harper AM, Jennet WB. Raised intracranial pressure and cerebral blood flow. 1. Cisterna magna infusion of primates. J Neurol Neurosurg Psychiatry. 1972;35:285-96.

20. Johnson IH, Rowan JO, Park DM, Reniie MJ. Raised intracranial pressure and cerebral bloob flow. 5 . Effects of episodic intracranial pressure waves in primaters. J Neurol Neurosurg Psychiatry. 1975;38:1076-82.

21. Juvela S, Heiskanen O, Poranen A, Valtonen S, Kuurne T, Kaste $\mathrm{M}$ et al. The treatment of spontaneous intracerebral hemorrhage. A prospective randomized trial of surgical and conservative treatment. J Neurosurg. 1989;70:755-8.

22. Kato Y, Auer LM. Cerebrovascular response to elevation of ventricular pressure. Acta Neurochir (Wien). 1989;98:184-8.

23. Langfitt TW, Weinstein JD, Kassel NF. Cerebral vasomotor paralysis produced by intracranial hypertensions. Neurology. 1965; 15:662-41.

24. Langfitt TW, Kassel NF, Weinstein JD. Cerebral blood flow with intracranial hypertension. Neurology. 1985;15:761-73.

25. Lassen NA. The luxury-perfusion syndrome and its possible relation to acute metabolic acidosis localized within the brain. Lancet. 1966;1:1113-5.

26. Lowell HM, Bloor BM. The effect of increased intracranial pressure on cerebrovascular hemodynamics. J Neurosurg. 1971;34:760-9.

27. Marshall LF, Durity F, Loundsbury R, Graham DI, Welsh F, Langfitt TW. Experimental cerebral oligoemia and ischemia produced by intracranial hypertension. Part 1. Pathofisiology, electrencefalography, cerebral blood flow, blood brain barrier and neurological function. Neurosurgery. 1975;43:308-17.

28. Marschall LF, Graham DI, Durity F, Lounsbury R, Welsh F, Langfitt TW. Experimental cerebral oligoemia and ischemia produced by intracranial hypertension. Part 2. Brain morphology. J Neurosurg. 1975;43:318-22.

29. Marshall LF, Welsh F, Durity F, Lounsbury R, Graham DI, Langfitt TW. Experimental cerebral aligoemia and ischemia produced by intracranial hypertension. Part 3 . Brain energy metabolism. J Neurosurg. 1975;43:323-8.

30. Matsumoto K, Hondo H. CT-guided stereotaxic evacuation of hypertensive intracerebral hematomas. J Neurosurg. 1984;61:440-8.

31. Mayer A, Rincon F. Treatment of intracerebral haemorrhage. Lancet Neurol. 2005;4:662-72.

32. Mayer SA, Sacco RL, Shi T, Mohr JP. Neurologic deterioration in noncomatose patients with supratentorial intracerebral hemorrhage. Neurology. 1994;44:1379-84.

33. Mayer SA, Brun NC, Begtrup K, Broderick J, Davis S, Diringer MN, et al. Recombinant activated factor VII for acute intracerebral hemorrhage. N Engl J Med. 2005;352:777-85.
34. Mckissock W, Richardson A, Taylot J. Primary intracerebral heamorrhage: a controlled trial of surgical and conservative treatment in 180 unselected cases. Lancet. 1961;2:221-226.

35. Mendelow AD, Gregson BA, Fernandes HM, Murray GD, Teasdale GM, Hope DT, et al. Early surgery versus initial conservative treatment in patients with spontaneous supratentorial intracerebral haematomas in the International Surgical Trial in Intracerebral Haemorrhage (STICH): a randomised trial. Lancet. 2005;365:387-97.

36. Misra UK, Kalita J, Ranjan P, Mandal SK. Manitol in intracerebral hemorrhage: a randomized controlled study. J Neurol Sci. 2005;234:41-5.

37. Naff NJ, Carhuapoma JR, Williams MA, Bhardwaj A, Ulatowski JA, Bederson J, et al. Treatment of intraventricular hemorrhage with urokinase: effects on 30-day survival. Stroke. 2000;31:841-7.

38. Naval NS, Nyquist PA, Carhuapoma JR. Management of spontaneous intracerebral hemorrhage. Neurosurg Clin North Am. 2008;19:415-23.

39. Park P, Fewel ME, Garton HJ, Thompson BG, Hoff JT. Recombinant active factor VII for the rapid correction of coagulopathy in nonhemophilic neurosurgical patients. Neurosurgery. 2003;53:34-8.

40. Qureshi Al, Tuhrim S, Broderick JP, Batjer HH, Hondo H, Hanley DF. Spontaneous intracerebral hemorrhage. N Engl J Med. 2001;344:1450-60.

41. Risberg J, Lundberg N, Ingva DM. Regional cerebral blood volume during acute transient rises of the intracranial pressure (plateau waves). J Neurosurg. 1969;31:303-10.

42. Symon K, Crockard HA, Juhasz J, Branstorn NM. The effect of intracranial hypertension on cerebrovascular resistance. An experimental study. Acta Neurochir (Wien). 1976;35:221-31.

43. Todd NV, Picozzi P, Crockard H, Rusel RWR. Recirculaton after cerebral ischemia:influence of duration of ischemia. Stroke. 1986;17:460-6.

44. Tuhrim S, Horowitz DR, Sacher M, Godbold JH. Valiation and comparison of models predicting survival following intracerebral hemorrhage. Crit Care Med. 1995;23:950-4.

45. Tuhrim S, Horowitz DR, Sacher M, Godbold JH. Volume of ventricular blood is an important determinant of outcome is supratentorial intracerebral hemorrhage. Crit Care Med. 1999;27:617-21.

46. Vespa P, McArthur D, Miller C, O'Phelan K, Frazee J, Kidwell $C$, et al. Frameless stereotactic aspiration and thrombolysis of deep intracerebral hemorrhage is associated with reduction of hemorrhage volume and neurological improvement. Neurocrit Care. 2005;2:274-81.

47. Wheelock B, Weir B, Watts R, Mohr G, Khan M, Hunter M, et al. Timing of surgery for intracerebral hematomas due to aneurysm rupture. J Neurosurg. 1983;58:476-81.

48. Xi G, Fewel ME, Hua Y, Thompson Jr. BG, Hoff JT, Keep RF. Intracerebral hemorrhage: pathophysiology and therapy. Neurocrit Care. 2004;1:5-18.

49. Xi G, Keep RF, Holf JT. Mechanisms o brain injury after intracerebral haemorrhage. Lancet Neurol. 2006;5:53-63.

50. Zwetnow N, Kjallquist A, Siesjo BK. Cerebral blood flow during intracranial hypertension in relation to tissue hypoxia and to acidosis in cerebral extracellular fluids. Prog Brain Res. 1968;30:87-92.

Endereço para correspondência

Marcos Augusto Stávale Joaquim

Alameda Campinas, $1.360,16^{\circ}$ andar

01404-002 - São Paulo, SP

Email:marcos.stavale@terra.com.br 